\title{
Displacement current induced by spin resonance in air-treated conjugated polymer diodes
}

Katsuichi Kanemoto, Hidenobu Matsuoka, Yujiro Ueda, Keisuke Takemoto, Keiji Kimura, and Hideki Hashimoto

\begin{tabular}{|c|l|}
\hline Citation & Physical Review B. 86(12); 125201 \\
\hline Issue Date & 2012-09-04 \\
\hline Type & Journal Article \\
\hline Textversion & Publisher \\
\hline Rights & $\begin{array}{l}\text { C2012 American Physical Society. This article may be downloaded for personal use only. } \\
\text { Any other use requires prior permission of the author and American Physical Society. } \\
\text { The following article appeared in Physical Review B Vol.86, Iss.12 and may be found at } \\
\text { https://doi.org/10.1103/PhysRevB.86.125201. }\end{array}$ \\
\hline DOI & \begin{tabular}{l} 
10.1103/PhysRevB.86.125201 \\
\hline
\end{tabular} \\
\hline
\end{tabular}

\author{
Self-Archiving by Author(s) \\ Placed on: Osaka City University
}

KANEMOTO, K., MATSUOKA, H., UEDA, Y., TAKEMOTO, K., KIMURA, K., \& HASHIMOTO, H. (2012). Displacement current induced by spin resonance in air-treated conjugated polymer diodes.

Physical Review B. 86. doi:10.1103/PhysRevB.86.125201 


\title{
Displacement current induced by spin resonance in air-treated conjugated polymer diodes
}

\author{
Katsuichi Kanemoto, ${ }^{1,}$ Hidenobu Matsuoka, ${ }^{1}$ Yujiro Ueda, ${ }^{1}$ Keisuke Takemoto, ${ }^{1}$ Keiji Kimura, ${ }^{1}$ and Hideki Hashimoto ${ }^{1,2}$ \\ ${ }^{1}$ Department of Physics, Osaka City University, 3-3-138 Sugimoto, Sumiyoshi-ku, Osaka 558-8585, Japan \\ ${ }^{2}$ CREST/JST and The OCU Advanced Research Institute for Natural Science and Technology (OCARINA), \\ Osaka City University, Osaka 558-8585, Japan
}

(Received 20 January 2012; revised manuscript received 24 May 2012; published 4 September 2012)

\begin{abstract}
We demonstrate that air-treated conjugated polymer diodes under photoexcitation give rise to a transient current exceeding a steady-state photocurrent by electron-spin resonance (ESR). The current induced by ESR is shown to exhibit a spiked time response for modulation of the resonance condition. The nature of the spike current is demonstrated to be a displacement current, which suggests that the spin transitions can change the film permittivity. This system has applications as devices enabling direct control of diode current by spin manipulation.
\end{abstract}

DOI: 10.1103/PhysRevB.86.125201

PACS number(s): 76.30.-v, 72.80.Le, 73.61.Ph

\section{INTRODUCTION}

Attempts to develop electronic devices that make use of the electron spin, referred to as spintronics, have given rise to a new, rapidly evolving field of science and technology. The central concept in spintronics is to utilize the interplay between the properties of electron spins and carriers (or current), which has led to the creation of several spin-based electronics. ${ }^{1-3}$ Such spin-based electronics have been developed primarily by utilizing magnetoresistance effects that indirectly convert a change in spin states into electronic signals via a magnetic field. In order to enhance the functionality of spintronics, techniques to more directly associate spin manipulation with electronics are required.

An electrically detected magnetic resonance (EDMR) technique has been developed to examine the spin properties of semiconductors ranging from inorganic $\mathrm{c}^{4,5}$ to organic semiconductors. ${ }^{6,7}$ The EDMR technique usually probes a small current induced by electron-spin resonance (ESR). In this study, we focus on the ESR-induced current because it directly associates the state of electron spin with electric signals and can be applied to spintronics. Such an ESR-induced current has indeed been employed to realize spin-based quantum devices such as spin resonance transistors, ${ }^{8,9}$ a spin memory, ${ }^{10}$ and spin-based quantum computers. ${ }^{11-14}$ In particular, a long spin coherence time in polymer semiconductors observed via the ESR-induced current by a pulsed EDMR technique has attracted much attention due to its potential capability for quantum information systems.$^{15}$ However, such ESR-induced currents are usually very small and typically much less than $1 \%$ of the steady-state current, which degrades their functionality as a spintronic system. Therefore, a new system of the ESR-induced current enabling much more efficient current generation is desired.

In this article, anomalous EDMR responses of conjugated polymer diodes are shown for poly[2-methoxy-5-(2'-ethylhexyloxy)-1,4-phenylene vinylene] (MEH-PPV) used as a prototypical polymer. MEH-PPV is one of the most studied materials for EDMR responses, ranging from $\mathrm{cw}-\mathrm{EDMR}^{6,16-18}$ to pulsed-EDMR experiments. ${ }^{7,15,19,20}$ We demonstrate that air treatment of the MEH-PPV diode remarkably enhances the EDMR response under photoexcitation. Such enhanced EDMR response is shown to arise from a temporal ESRinduced current occurring due to a change in the polarization field within the diode. This finding can be applied to developments of new devices enabling direct control of device current by spin transitions.

\section{EXPERIMENT}

The MEH-PPV diodes were fabricated by spin casting MEH-PPV dissolved in chlorobenzene onto an indium-tinoxide (ITO)-coated glass substrate (anode) and the subsequent deposition of an $\mathrm{Al}$ electrode by vacuum evaporation (cathode). We prepared two types of MEH-PPV diodes differing in the time of air (or water) exposure. One was a diode fabricated under conditions to minimize air and water exposure time as best as we could by utilizing a glove box and a dehydrated solvent, termed a pristine diode. The other was fabricated under air exposure and a nondehydrated solvent, termed the air-treated diode. The thickness of the polymer layer was about $100 \mathrm{~nm}$ and the active area of the diode was $2 \mathrm{~mm} \times 3 \mathrm{~mm}$. The diodes were loaded into a 5-mm-diameter glass tube for EDMR measurements.

The EDMR experiments were carried out under photoexcitation with no applied bias (short circuit condition) using an $\mathrm{X}$-band ESR spectrometer by modulating a microwave power with a PIN modulator $(1 \mathrm{kHz})$. A diode-pumped solid-state (DPSS) laser with cw 473-nm output (CNI) was used for photoexcitation. The EDMR signals were amplified with a current amplifier (Stanford Research Systems SR570). EDMR spectra were measured by recording the modulation current using a lock-in amplifier (SR830). The time response of the EDMR signals was measured using a digital oscilloscope for modulated resonant microwave power. The signals were recorded through an input resistance of $1 \mathrm{M} \Omega$, suggesting that actual time responses can be more rapid than the observed time responses shown in the following figures. The irradiation of high-power microwave sometimes yielded non-negligible background current probably due to the electric field component of the microwave or a heating effect by the microwave. The EDMR signals were thus obtained by subtracting the background signals measured at an off-resonance magnetic field. All EDMR measurements were performed at room temperature under $\mathrm{N}_{2}$ gas flow to minimize interactions with oxygen that could affect the paramagnetic spins in the film. ${ }^{21}$ 

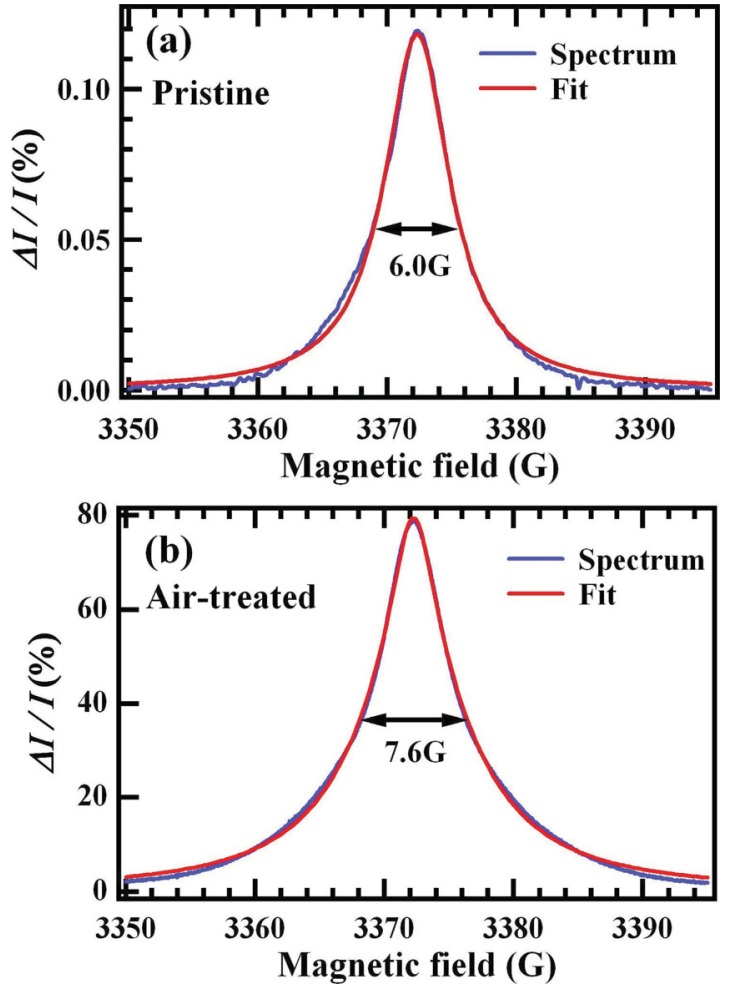

FIG. 1. (Color online) Electrically detected magnetic resonance (EDMR) spectra of (a) pristine and (b) air-treated MEH-PPV diodes measured with a lock-in technique. The intensity of the EDMR signal $(\Delta I)$ is normalized against that of the steady-state photocurrent measured simultaneously $(I)$. The microwave power was $200 \mathrm{~mW}$. The photoexcitation intensity was 20 and $22 \mathrm{~mW} / \mathrm{cm}^{2}$ for the pristine and air-treated diodes, respectively. The value of the steady-state photocurrent in the pristine diode was approximately $200 \mathrm{nA}$. Results of spectral fit using a Lorentzian line shape are also shown for EDMR spectra of both the pristine and air-treated diodes.

\section{RESULTS AND DISCUSSION}

The EDMR spectrum under photoexcitation for the pristine MEH-PPV diode is shown in Fig. 1(a). The $g$ value determined from the peak position was approximately 2.003 , indicating that the origin of the signals is $\pi$ electrons of MEH-PPV. The intensity of the EDMR signal was normalized with respect to the simultaneously measured steady-state photocurrent (PC). The peak intensity of the signals is approximately $0.1 \%$, which is almost consistent with previous reports on the EDMR intensity of MEH-PPV diodes. ${ }^{18}$ In contrast, following exposure to air, the EDMR signals obviously became enhanced. Figure 1(b) shows the EDMR spectrum of the air-treated MEH-PPV diode. The air-treated diode exhibits a peak response as high as $80 \%$, which is almost comparable with its steady-state PC. This response is surprising because, to our best knowledge, the largest EDMR response ever reported is $10 \%$, which was obtained from an unsubstituted PPV diode under photoexcitation. ${ }^{22}$

Comparison of the EDMR spectrum for the pristine and air-treated diodes indicates that the peak positions of the spectra are close to each other. However, there exist small differences in the spectral line shape and the linewidth between the two spectra. It is indeed shown that the full width at half

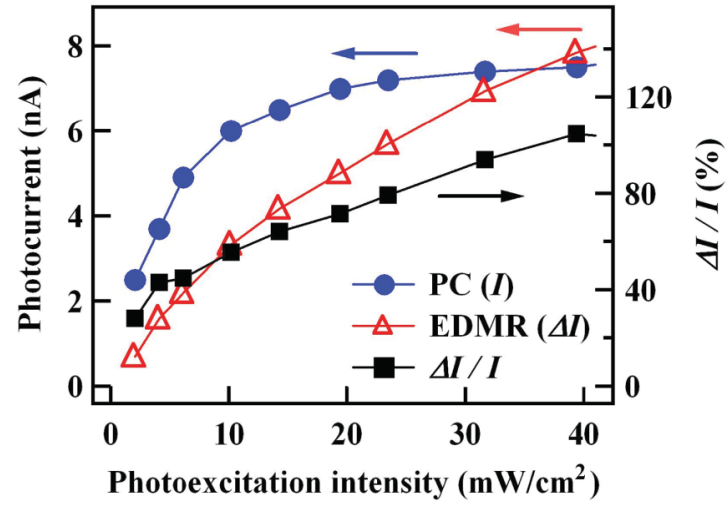

FIG. 2. (Color online) Photoexcitation intensity dependence of the steady-state photocurrent (PC), the EDMR signals $(\Delta I)$, and the normalized EDMR response $(\Delta I / I)$ for the air-treated $\mathrm{MEH}$ PPV diode. The EDMR signals were recorded at $3372 \mathrm{G}$. The lines connecting the data points were added as visual guides.

maximum (FWHM) of the air-treated diode $(7.6 \mathrm{G})$ is larger than that of the pristine diode $(6.0 \mathrm{G})$. Figures 1(a) and 1(b) also show results of spectral fit using Lorentzian curves for the EDMR spectra. The spectrum of the pristine diode was nearly reproduced by a single Lorentzian curve as shown in the figure, whereas that of the air-treated diode was well reproduced by the sum of two Lorentzian curves with the same center field whose FWHMs were 4.0 and 11.4 G. From this analysis, we conclude that the spectrum of the air-treated diode consists of the sum of a quasi-Lorentzian component responsible for the pristine diode and additional spin components, which may cause the broader width in the air-treated diode.

Figure 2 shows the photoexcitation intensity dependence of the steady-state PC, the absolute EDMR intensity, and the normalized EDMR intensity measured at the resonance peak $(3372 \mathrm{G})$ for the air-treated diode. Both of the absolute EDMR and steady-state PC signals simply increase with increasing photoexcitation intensity due to an increase in the number of absorbed photons. However, the steady-state PC is somewhat saturated above $10 \mathrm{~mW} / \mathrm{cm}^{2}$, while the EDMR signal continues to increase in the same region. Related to this trend in the EDMR signal, the normalized EDMR intensity increases almost linearly and finally attained $100 \%$ at $40 \mathrm{~mW} / \mathrm{cm}^{2}$ photoexcitation. This indicates that strong photoexcitation is needed to obtain a large normalized EDMR response. In addition, the behavior of saturation in the steady-state PC indicates that mobile photocarriers are not proportionally generated for the increase in photoexcitation. This suggests that secondary species such as trapped carriers or spin pairs should be generated at this photoexcitation. Therefore, such secondary species as well as photocarriers are considered to contribute to the enhanced EDMR signals.

The large EDMR signal comparable with the steady-state PC suggests that the EDMR signal might be detectable as a steady-state current without using a lock-in technique for microwave modulation. However, no EDMR signals were obtained when the resonant microwave was continuously irradiated. The time response of the EDMR signals was then examined to elucidate the origin of the large EDMR signals. Figures 3(a) and 3(b) present the time responses of EDMR 

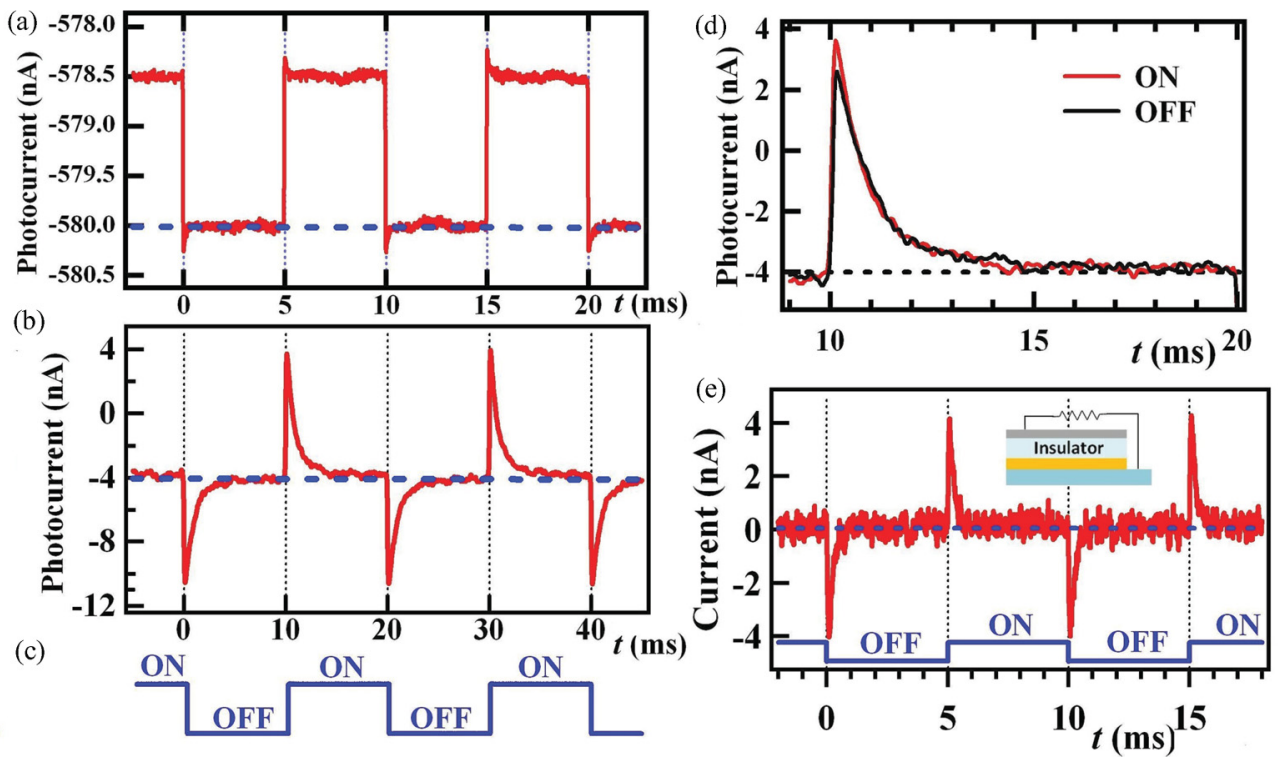

FIG. 3. (Color online) Time response of photocurrent under resonance condition (3372 G) for the (a) pristine and (b) air-treated MEH-PPV diodes when repeatedly switching the ESR-resonant microwave power on and off, as shown in (c). The horizontal broken lines in (a) and (b) represent a steady-state photocurrent. The intensity of the photoexcitation $\left(I_{p}\right)$ was $22 \mathrm{~mW} / \mathrm{cm}^{2}$. (d) Comparison of the time trace at the moment of the on- and off-microwave irradiation in (b). The trace of the off-irradiation was inverted and set so that its initial point was in accord with that of the on-irradiation. (e) Time response of the peak EDMR signal for an ITO/air-treated MEH-PPV/Zeonex (200 nm)/Al device $\left(I_{p}=20 \mathrm{~mW} / \mathrm{cm}^{2}\right)$.

signals measured at the resonance peak (3372 G) for the pristine and air-treated MEH-PPV diode, respectively. In these measurements, the microwave power was repeatedly switched on and off under the resonance condition as shown in Fig. 3(c). Figure 3(a) demonstrates that the pristine diode exhibits a squared time response of decreasing photocurrent for the squared ESR modulation. In the mechanisms of EDMR that have been proposed for MEH-PPV, ${ }^{15,18,23}$ the presence of the ESR-induced current that steadily responds to the variation of the ESR condition was assumed. The result in Fig. 3(a) is consistent with the mechanisms. In contrast, the time response for the air-treated diode exhibits positive and negative spike currents, when the microwave power is switched on and off, respectively. Moreover, it should be mentioned that the spike current upon ESR greatly exceeds the steady-state PC.

To our best knowledge, an EDMR time response exhibiting a spiked current has never been reported. The EDMR phenomenon observed in this study is thus regarded to be caused by an EDMR mechanism essentially different from previous reports. The observed spike response is typical behavior of a displacement current (or a polarization current), which is induced by a change in the polarization electric field within the film and flows between two electrodes outside the film. ${ }^{24}$ If this is the case, traces of the time response should be similar for the on- and off-microwave irradiation. Figure 3(d) compares the time traces at the moment of the on- and off-microwave irradiation and indicates that the two traces are similar except for the peak intensity, which could be due to carrier quenching at the electrode-polymer interfaces.

Additionally, in the case of the displacement current, current spikes should be observable even in a system containing an insulating layer, because the displacement current essentially occurs regardless of injection or emission of charge for the film. We thus performed EDMR measurements for an ITO/air-exposed MEH-PPV/cyclo-olefin polymer (Zeonex) $(200 \mathrm{~nm}) / \mathrm{Al}$ device where the Zeonex layer is used as an insulating layer. The insulating device yielded EDMR signals at the same magnetic field position as the MEH-PPV diode; the actual time response of the EDMR signals of the insulating device is shown in Fig. 3(e). It demonstrates that current spikes are still observed despite a negligibly small steady-state PC due to the presence of the insulating layer. It is thus concluded that the displacement current is the origin of the observed current spikes and that the enhanced EDMR response in the air-treated diode was given by the displacement current.

A displacement current is usually generated outside a diode so that it retains the potential difference between the two electrodes. Also, the direction of the displacement current is uniquely determined depending on whether the polarization electric field inside the diode increases or decreases for an event. Under these considerations, we propose a mechanism for the observed spike current as illustrated in Fig. 4. The polarization field is formed in the direction antiparallel to the built-in electric field that is directed from the $\mathrm{Al}$ to ITO electrodes due to the difference in the work function. Under this condition, we expect the ESR transition to decrease the concentration of mobile carriers forming the polarization field, and this decrease of the carriers temporarily reduces the carrier polarization field, which leads to generation of the displacement current in the direction from Al to ITO. In the case of the off-spin transition, the decreased carrier concentration is recovered, which generates displacement current from ITO to Al. This is a possible mechanism for the observed current spikes.

The detailed mechanism of the ESR-induced decrease in the mobile carrier concentration is not yet completely understood 


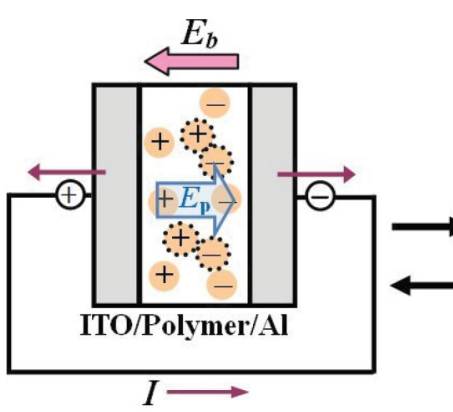

ESR OFF

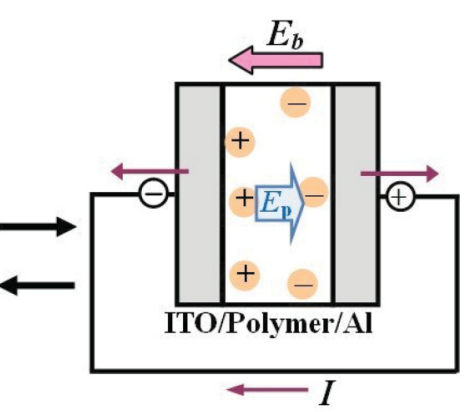

ESR ON
FIG. 4. (Color online) Schematic of a possible mechanism for the current spikes. It is assumed that a polarization electric field $\left(E_{p}\right)$ by mobile carriers is antiparallel to the built-in field $\left(E_{b}\right)$ formed in the direction from the Al to the ITO electrodes. Mobile carriers represented by dotted circles disappear as a result of ESR and thereby the polarization field is reduced. A spiked displacement current is then generated outside the diode to retain the potential difference. When the ESR transition is switched off, an opposite displacement current is generated to recover the reduced polarization electric field.

at the present stage. However, trapped carriers or spin pairs have been suggested above to be related to the enhanced EDMR signals. Moreover, the participation of spin pairs has been proposed in the EDMR mechanism for the photoexcited MEH-PPV. ${ }^{15,18,20,24}$ Therefore, the decrease (or increase) of the mobile carrier concentration would be caused by formation (or dissociation) of spin pairs induced by the ESR transition. Also, the air exposure for the MEH-PPV diode is expected to generate carrier-trap sites within the diode or at the interface between the polymer and the electrodes. We thus consider that the polarization electric field leading to the spike current is formed by mobile photocarriers trapped at carrier-trap sites.

The significance of the observations in this study is that the obtained ESR-induced current, comparable to the steady-state PC, enables direct control of device current by spin manipulation, without drawing small signals out of a large background steady-state current. This enhances the functionality of the ESR-induced current for applications to spintronics devices. In particular, we observed a strong EDMR response also by modulating the resonant magnetic field under continuous microwave irradiation. Therefore, a magnetic field as well as a microwave can be used as elements to control the ESR-induced current.
Although the polarization field was suggested above to vary for generation of the spike current, such a variation in the polarization field gives rise to a change in the film permittivity (or capacitance). In this sense, the origin of the ESR phenomena with a spike current is similar to that of the capacitance change induced by ESR identified for materials. ${ }^{25,26}$ We here estimate the proportion of the change of the permittivity induced by the ESR transition $\Delta \varepsilon / \varepsilon$, where $\varepsilon$ is the film permittivity. Assuming that the change of the charge per square meter, $\Delta Q$, which is calculated by integrating the polarization current density, is represented by $\Delta C V_{b}$ where $C$ is the capacitance and $V_{b}$ is the built-in voltage, $\Delta \varepsilon / \varepsilon$ can be estimated by the relation $d \Delta Q /\left(\varepsilon V_{b}\right)$ where $d$ is the film thickness. $\Delta Q$ was calculated to be $1.4 \times 10^{-6} \mathrm{C} \mathrm{m}^{-2}$ from the result in Fig. 3(b), which yields $\Delta \varepsilon / \varepsilon \approx 1 \%$ for $\varepsilon=3$ and $V_{b}=0.5 \mathrm{~V}$ obtained from the work function difference between ITO $(4.8 \mathrm{eV})$ and $\mathrm{Al}(4.3 \mathrm{eV})$.

We finally note that a similar spiked strong EDMR response was observed also from diodes of regioregular poly(3-hexylthiophene) and of $\mathrm{C}_{60}$. The observed EDMR features in the present study would thus be common features in organic semiconductors. Therefore it is expected that the selection or development of optimized organic semiconductors further improves performance of the ESR-induced current.

\section{CONCLUSIONS}

In summary, we have demonstrated that the polymer diode examined in this study exhibits a large ESR-induced current under photoexcitation after air treatment of the diode. The ESR current was shown to exhibit a spiked time response for modulation of the resonance condition. The spike current greatly exceeded the steady-state PC. The nature of the ESR current was shown to be a displacement current caused by a change in the diode permittivity. This effect is applicable to the construction of spin-electronic devices.

\section{ACKNOWLEDGMENTS}

We thank T. Suzuki of JEOL for helpful discussions. This work was supported in part by a Grant-in-Aid (Grant No. 10016820) from the Japanese Ministry of Education, Culture, Sports, Science, and Technology. K.K. acknowledges funding from the CASIO Science Promotion Foundation. H.H. thanks the Nissan Foundation and HFSP for financial support.

\footnotetext{
*Author to whom correspondence should be addressed: kkane@sci.osaka-cu.ac.jp

${ }^{1}$ G. A. Prinz, Science 282, 1660 (1998).

${ }^{2}$ S. A. Wolf, D. D. Awschalom, R. A. Buhrman, J. M. Daughton, S. von Molnár, M. L. Roukes, A. Y. Chtchelkanova, and D. M. Treger, Science 294, 1488 (2001).

${ }^{3}$ S. D. Bader and S. S. P. Parkin, Annu. Rev. Condens. Matter Phys. 1, 77 (2010).

${ }^{4}$ M. Stutzmann, M. S. Brandt, and M. W. Bayerl, J. Non-Cryst. Solids 266-269, 1 (2000).
}

${ }^{5}$ P. M. Lenahan and J. F. Conley, IEEE Trans. Dev. Mater. Reliab. 5, 90 (2005)

${ }^{6} \mathrm{~J}$. Shinar, in Handbook of Organic Conductive Molecules and Polymers, edited by H. S. Nalwa (John Wiley \& Sons, Sussex, UK, 1997), Vol. 3, p. 319.

${ }^{7}$ C. Boehme, and D. R. McCamey, in Organic Spintronics, edited by Z. V. Vardeny (CRC Press, Boca Raton, FL, 2010), p. 257.

${ }^{8}$ R. Vrijen, E. Yablonovitch, K. Wang, H. W. Jiang, A. Balandin, V. Roychowdhury, T. Mor, and D. DiVincenzo, Phys. Rev. A 62, 012306 (2000). 
${ }^{9}$ M. Xiao, I. Martin, E. Yablonovitch, and H. W. Jiang, Nature 430, 435 (2004).

${ }^{10}$ D. R. McCamey, J. Van Tol, G. W. Morley, and C. Boehme, Science 3301652 (2010).

${ }^{11}$ F. H. L. Koppens, C. Buizert, K. J. Tielrooij, I. T. Vink, K. C. Nowack, T. Meunier, L. P. Kouwenhoven, and L. M. K. Vandersypen, Nature 442, 766 (2006).

${ }^{12}$ A. R. Stegner, C. Boehme, H. Huebl, M. Stutzmann, K. Lips, and M. S. Brandt, Nat. Phys. 2, 835 (2006).

${ }^{13}$ G. W. Morley, D. R. McCamey, H. A. Seipel, L.-C. Brunel, J. van Tol, and C. Boehme, Phys. Rev. Lett. 101, 207602 (2008).

${ }^{14}$ A. R. Stegner, R. N. Pereira, K. Klein, R. Lechner, R. Dietmueller, M. S. Brandt, M. Stutzmann, and H. Wiggers, Phys. Rev. Lett. 100, 026803 (2008).

${ }^{15}$ D. R. McCamey, H. A. Seipel, S. Y. Paik, M. J. Walter, N. J. Borys, J. M. Lupton, and C. Boehme, Nat. Mater. 7, 723 (2008).

${ }^{16}$ G. B. Silva, L. F. Santos, R. M. Faria, and C. F. O. Graeff, Physica B 308-310, 1078 (2001).

${ }^{17}$ F. A. Castro, G. B. Silva, L. F. Santos, R. M. Faria, F. Nüesch, L. Zuppiroli, and C. F. O. Graeff, J. Non-Cryst. Solids 338-340, 622 (2004).
${ }^{18}$ F. Wang, C. G. Yang, E. Ehrenfreund, and Z. V. Vardeny, Synth. Met. 160, 297 (2010).

${ }^{19}$ D. R. McCamey, K. J. van Schooten, W. J. Baker, S. Y. Lee, S. Y. Paik, J. M. Lupton, and C. Boehme, Phys. Rev. Lett. 104, 017601 (2010).

${ }^{20}$ J. Behrends, A. Schnegg, K. Lips, E. A. Thomsen, A. K. Pandey, I. D. W. Samuel, and D. J. Keeble, Phys. Rev. Lett. 105, 176601 (2010).

${ }^{21}$ K. Kanemoto and J. Yamauchi, J. Phys. Chem. B 105, 2117 (2001).

${ }^{22}$ V. Dyakonov, N. Gauss, G. Rosler, S. Kargs, and W. Riess, Chem. Phys. 189, 687 (1994).

${ }^{23}$ D. R. McCamey, S. Y. Lee, S. Y. Paik, J. M. Lupton, and C. Boehme, Phys. Rev. B 82, 125206 (2010).

${ }^{24}$ K. C. Kao, Dielectric Phenomena in Solids (Elsevier Academic Press, Amsterdam, 2004).

${ }^{25}$ M. S. Brandt, R. T. Neuberger, M. W. Bayerl, and M. Stutzmann, Jpn. J. Appl. Phys. 38, L1172 (1999).

${ }^{26}$ M. S. Brandt, R. T. Neuberger, and M. Stutzmann, Appl. Phys. Lett. 76, 1467 (2000). 